\title{
William Osler: el hombre y sus descripciones
}

\author{
PABLO YOUNG ${ }^{1}$, BÁRBARA C. FINN ${ }^{1}$, JULIO E. BRUETMAN ${ }^{1}$, \\ JOHN D. C. EMERY ${ }^{1}$, ALFREDO BUZZI ${ }^{2}$
}

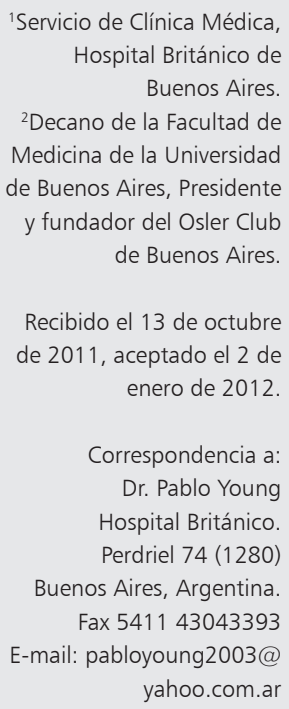

\section{William Osler (1849-1919): the man and his descriptions}

William Osler was generally regarded as the greatest and most respected physician of his time. This paper describes Osler's life, his philosophy and views. He was an outstanding clinician who emphasized bedside teaching and observation. He possessed an extraordinary charm that inspired many others. As Professor of Medicine at four institutions in three countries, he was a great influence on medical education. He was a prolific writer, and his textbook became the most popular and widely read treatise on medicine in the world. He also was a medical historian, a classical scholar, and an avid bibliophile. He emphasized the value of hard work and ongoing education. His compassion and concern for patients and colleagues reflected his personality. We summarize Osler's descriptions, and some of his aphorisms. His wisdom is as relevant now, as it was in his time. Osler blended the art and science of Medicine perhaps better than anyone else, and remains a valuable role model for students and physicians more than ninety two years after his death.

(Rev Med Chile 2012; 140: 1218-1227).

Key words: Aphorisms and proverbs; History of Medicine; Portraits.
W illiam Osler nació el 12 de julio de 1849 en la población de Bond Head, provincia de Ontario, Canadá ${ }^{1-6}$. Sir William Osler (Figura 1) ha sido el médico más influyente del siglo XX. De él se ha dicho que sirvió a tres naciones (Canadá, Estados Unidos de Norteamérica e Inglaterra) y las tres lo tuvieron por hijo.

Entre otros, el legado de Osler fue: 1) la enseñanza al lado del paciente; 2) el nuevo currículo, la educación médica y la promoción de la investigación en los estudiantes; 3) el conocimiento de la historia y el humanismo y 4) el amor a la medicina interna ${ }^{1-6}$.

Osler no fue un innovador, pero sí un erudito y un magnífico clínico, observador atento y descriptor de la evolución natural de las enfermedades. Fue un gran profesor y un gran maestro. Quizás su atractivo más relevante residió en el encanto singular de su presencia, en la brillantez de su intelecto, en la belleza de su carácter, a veces melancólico y de su vida, y en el ejemplo que fue para sus colegas y estudiantes.

\section{Su Familia}

Era el octavo hijo de la familia compuesta por Featherstone Lake Osler (1805-1895) y Ellen Pickton Osler (1806-1906). Sus padres, galeses de origen celta, viajaron desde Gran Bretaña a Canadá como misioneros anglicanos. El nombre de William le fue impuesto en honor al rey William III de Inglaterra. Se casó el 15 de mayo de 1892 con la viuda de un cirujano de Philadelphia, el Dr. Samuel W. Gross, la Sra. Grace Revere Gross, de 37 años y nieta del famoso patriota Paul Revere que se destacó durante la Guerra de la Independencia de Estados Unidos de Norteamérica. El primer hijo murió a los pocos días de haber nacido. Posteriormente, tuvieron otro hijo, Edward Revere 


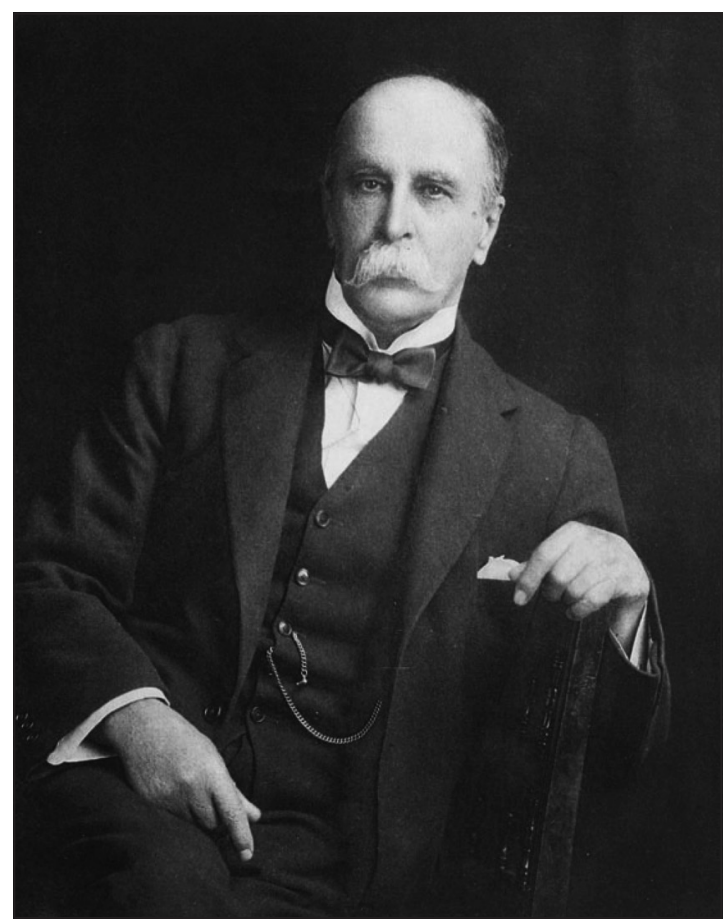

Figura 1. Dr. Sir William Osler.

Osler, quien nació el 28 de diciembre de 1895 en Baltimore (Figura 2). Dos meses antes de su matrimonio, el 24 de febrero de 1892, y casi dos años después de haber empezado a trabajar en septiembre de 1890, le entregó a su novia uno de sus legados más importantes "Los principios y práctica de la medicina" y con la entrega de éste, le propuso matrimonio ${ }^{6}$.

\section{Su vida y estudios}

En 1866, a los 17 años, ingresó en el Trinity College de Weston. Aunque inicialmente orientado por su padre a seguir una carrera clerical, se decidió por la Medicina e ingresó en 1870, a los 21 años, la Escuela de Medicina de Toronto. Dos años después, en 1872, recibió su título de médico en la Universidad de McGill en Montreal ${ }^{7}$. Amplió sus conocimientos en la Universidad de Londres, donde estudió fisiología entre 1872 y 1873, y los completó en Berlín, donde conoció a Rudolf Virchow (1821-1902). En Berlín y Viena, entre los años 1873 y 1874, llevó a cabo su formación de clínica en los hospitales ${ }^{1,2}$.

En 1874 regresó a Canadá en calidad de docen-

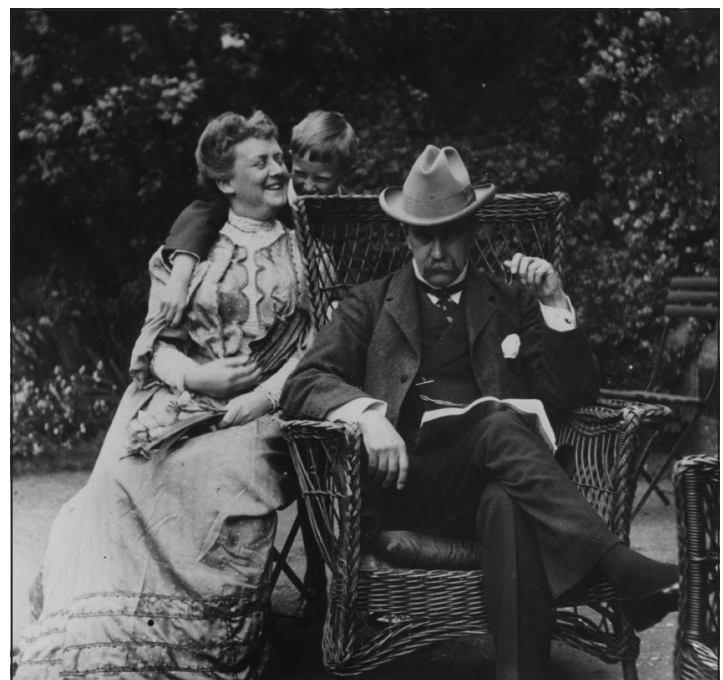

Figura 2. William Osler con su esposa e hijo.

te (Lecturer) de la Universidad de McGill' . En 1875, contando con 26 años de edad, fue Profesor en la misma Universidad, y un año después, en 1876, trabajó como patólogo en el Hospital General de Montreal. En 1878 aceptó el puesto de doctor en el Hospital General de Montreal. Aprendió en esa época de Arthur Conan Doyle y del maestro de éste, Joseph Bell (1837-1911), el método de Zadig, que fue tomado de "Zadig or Destiny", una novela escrita en 1747 por François Marie Arouet (16941778), más conocido como Voltaire. Zadig tenía una afinidad por la naturaleza y por cada detalle trivial, lo que utilizaba para realizar descripciones majestuosas, como Sherlock Holmes. Este método, pensaba Osler, era importante para aplicarlo en el diagnóstico médico ${ }^{1-8}$.

En 1881, en un congreso internacional en Londres, conoció a John Shaw Billings (1838-1913), el organizador de todas las bibliotecas médicas de Estados Unidos de Norteamérica y fundador en 1865 de la Biblioteca Nacional de Medicina, en Bethesda. Con él estableció contacto para proveer de material la Biblioteca del Cirujano General, y luego mantuvo su amistad y el trabajo en conjunto cuando comenzó a trabajar en la Universidad Johns Hopkins.

En 1884, a los 35 años de edad, dejó Canadá para establecerse en Filadelfia, Estados Unidos de Norteamérica, al ser invitado para hacerse cargo de la Cátedra de Medicina Clínica de la Universidad de Pennsylvania, la más antigua de los Estados 
Unidos de Norteamérica ${ }^{1-6}$. En 1889, contando con 40 años, fue contratado como Profesor de Medicina de la Universidad Johns Hopkins, siendo el primer profesor, además de Jefe de Servicio de Medicina Interna del mismo hospital. En este Hospital realizó una tarea memorable para desarrollar la enseñanza científica de la medicina interna en las salas de internación ${ }^{4-6}$. En 1893, a los 44 años de edad, inauguró la Escuela de Medicina del Johns Hopkins, contribuyendo a su liderazgo, junto a personalidades médicas como William Henry Welch (1856-1934), patólogo, William Stewart Halsted (1852-1922), cirujano, y Howard Atwood Kelly (1857-1943), ginecológo. Juntos constituyeron "los cuatro grandes del Hospital Johns Hopkins" retratado en el famoso cuadro de John Singer Sargent (1856-1925)(Figura 3) ${ }^{3-6}$.

Consideró como sus mentores a William A. Jonson, párroco de Weston Ontario, James Bovell de la Escuela de Medicina de Toronto y Robert Palmer Howard, decano y profesor de medicina de la Universidad de McGill, Montreal. Uno de los libros que recomendaba el reverendo Jonson era "Religio Medici", escrito en 1642 por el famoso

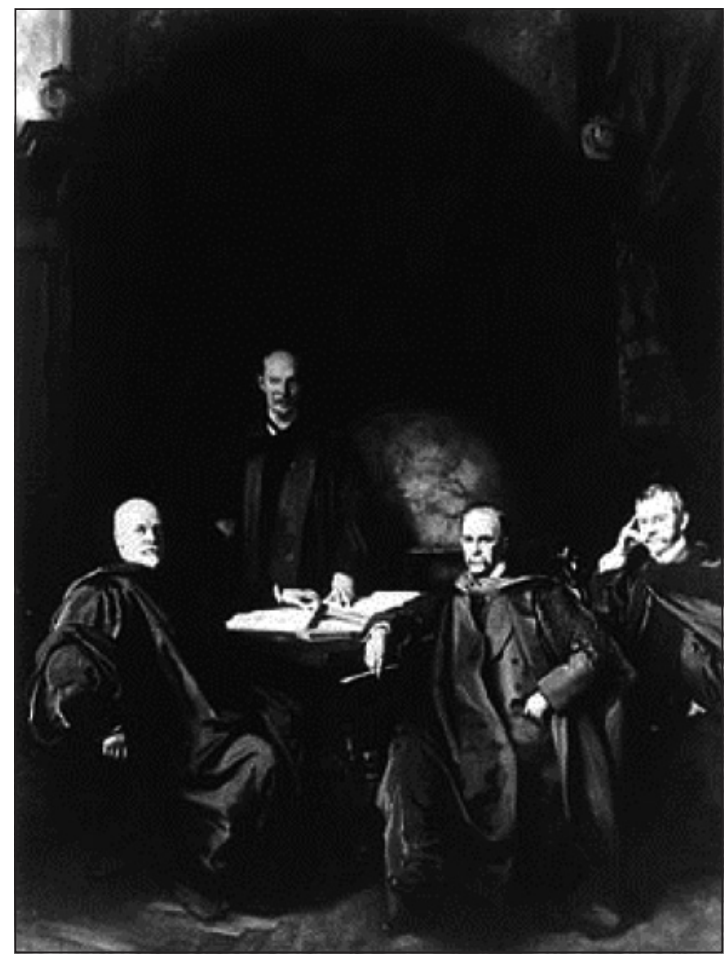

Figura 3. Los cuatro médicos por John Singer Sargent, 1905. médico Sir Thomas Brown (1605-1682); fue el primer libro que compró Osler, y según él "este libro era su amigo", lo tenía siempre sobre su mesa de luz, y lo citaba frecuentemente.

Basando su experiencia en los diferentes centros hospitalarios y en las universidades en las que ejerció la docencia, contribuyó a diseñar el currículo que integraba la formación hospitalaria con los estudios de medicina: la estructura actual de la formación médica se basa en las innovaciones que introdujo en la Universidad de Johns Hopkins, donde impuso la modalidad de "la enseñanza al lado de la cama del paciente" (Figura 4), tendencia iniciada en el siglo XVI por Giambatista da Monte (1498-1551) integrante de la primera Escuela Italiana y continuada, entre otros, por Hermann Boerhaave (1668-1738) en Leyden, Holanda, y Ferdinand Ritter von Hebra (1816-1880) y Moritz Kaposi (1837-1902) dermatólogos de la segunda escuela vienesa en Austria, hacia 1845, donde Osler lo aprendió, y lo puso en práctica en McGill, Filadelfia. Este último lo institucionalizó en las prácticas universitarias en el Johns Hopkins en 1914.

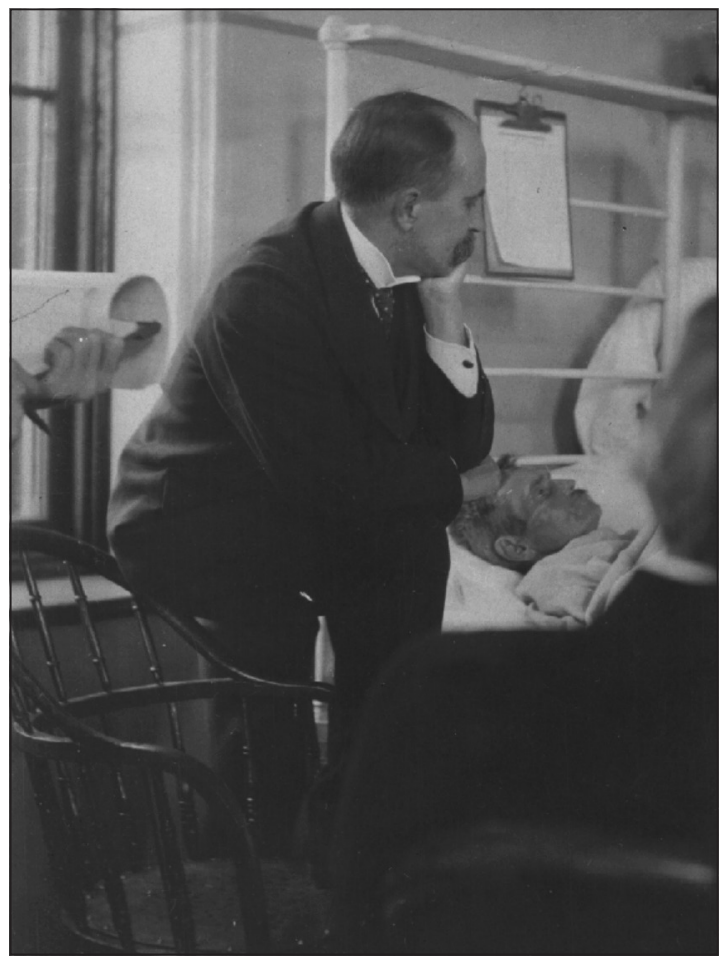

Figura 4. Osler al lado de la cama del paciente. 
Defendió la práctica de la autopsia, pues consideró que el examen post-mortem es importante para reconocer los aciertos o las equivocaciones en el diagnóstico. Se dice que personalmente practicó cerca de mil autopsias 9 . Defendió la teoría del "unicismo", que trata de explicar los síntomas y signos de los pacientes con un único diagnóstico, lo cual se contrapone con la llamada sentencia de Hickam $^{10}$. No se conocían por la época los estudios aleatorizados prospectivos, ni los controlados, ni los de análisis de casos, ni los metaanálisis, y así sus enseñanzas se basaban en la cuidadosa observación clínica y la gran experiencia y conocimiento de las enfermedades.

En 1904 se trasladó a Inglaterra, a la Universidad de Oxford, donde fue nombrado Profesor Regio o Real de medicina (catedrático designado directamente por el rey o la reina), que ocupó con brillantez durante 14 años. Estando allí vivió la tragedia de la muerte de su hijo, de 21 años, que supo sobrellevar con dignidad y resignación ${ }^{11}$. Este hijo era teniente del cuerpo expedicionario británico durante la primera Guerra Mundial, y en la batalla de Ypres, Bélgica, falleció tras sufrir heridas graves en el tórax y abdomen por esquirlas de granada.

Definitivamente Osler había estudiado medicina influenciado por la Escuela de Edimburgo y Glasgow. En efecto, la Facultad de Medicina de McGill, su alma mater, fue fundada en 1821 primordialmente por escoceses, marcados con el sello indeleble de los grandes médicos fisiólogos, anatomistas y cirujanos, John (1728-1793) y William Hunter (1718-1783) ${ }^{3-6}$. Osler conocíó ampliamente la medicina inglesa que se practicaba y enseñaba en los hospitales Guy's, St. Bartholomew, St. Thomas y en el Hospital General de Londres, que había visitado en 1874. Tampoco desconoció las virtudes de la medicina alemana y francesa, pues estuvo familiarizado con las obras de Rokitansky y las del famoso René Laënnec. Ante la Academia de Medicina de Nueva York alguna vez dijo: "En el método de enseñanza que puede llamarse natural, el estudiante comienza con el enfermo, continúa con el enfermo y termina sus estudios con el enfermo, utilizando conferencias como herramientas y como medios que conducen a su fin. Enséñeles el modo de observar, suminístreles suficientes hechos qué observar, y así las lecciones saldrán de los hechos mismos"1-6.

También afirmaba que "la medicina es una ciencia de probabilidades y un arte de manejar la incertidumbre". Es muy seguro que si Osler estuviese vivo hoy, principios del siglo XXI, estaría de acuerdo con D.L. Sackett, uno de los pontífices de la medicina basada en la evidencia, quien en 1996 notaba que esta nueva estrategia es la integración del saber y la experiencia médica, con lo mejor de la evidencia disponible en la literatura científica, y que tal evidencia jamás reemplazará a la habilidad y la experiencia clínica.

\section{Sus intereses y aportes}

Sus contribuciones a la medicina incluyen sus investigaciones sobre la fiebre tifoidea, la malaria, la neumonía, la amebiasis, la tuberculosis, y la litiasis renal, cuyo cuadro clínico describió magistralmente, y padeció en dos ocasiones ${ }^{12}$. Fue quien postuló la posibilidad de que el asma sea secundario a reflujo gastroesofágico, lo que llamó, asma gástrica ${ }^{13}$. En 1888 describió la entidad clínica denominada "edema angioneurótico"en su variante hereditaria, cuya variante adquirida había descripto Quincke en 1882. En 1896, dio a conocer un tratamiento de la enfermedad de Addison con extractos adrenales frescos de cerdos y en 1900 publicó junto al patólogo canadiense John McCrae (1872-1915), su experiencia clínica en 150 pacientes con cáncer gástrico. Describió en 1895 las complicaciones viscerales del lupus eritematoso diseminado. Fue condecorado por la Corona Británica con el título de Sir en 1911 por sus grandes contribuciones en el campo de la medicina.

Distintas enfermedades y síntomas llevan su nombre (Tabla 1). El signo o maniobra de Osler se utiliza para detectar la pseudohipertensión, que es cuando la presión sanguínea medida con el esfingomanómetro es artificialmente alta, debido a la calcificación de la pared arterial. La maniobra es positiva si el pulso a la palpación persiste, aún si no se escuchan latidos con el estetoscopio, luego de inflar el manguito del esfigmomanómetro por encima de la presión sistólica. Los nódulos de Osler son nódulos subcutáneos, dolorosos, ubicados habitualmente en extremidades, y se observan en la endocarditis. Cuando estos son de mayor extensión, se conocen como placa de Deloré, y están presentes por 2 a 6 días.

Además describió, junto a Jaccoud y Libman, y luego de una detallada observación de 200 
Tabla 1. Cronología de las descripciones de William Osler

\begin{tabular}{|c|c|}
\hline Año & Descripción de enfermedad, síndrome, signo o fenómeno \\
\hline 1873 & Describió las plaquetas y el fenómeno de Osler o aglutinación plaquetaria \\
\hline 1875 & Describió un parásito nematodo conocido como filaria de Osler \\
\hline 1876 & Describió la fagocitocis antes que Elie Metchnikoff \\
\hline 1885 & Enfermedad de Osler-Libman-Sacks o endocarditis verrugosa en LES \\
\hline 1885 & Enfermedad de Jaccoud-Osler-Libman o endocarditis bacteriana \\
\hline 1885 & Describió los aneurismas micóticos en la endocarditis \\
\hline 1885 & Describió la facie maligna lenta de Jaccoud-Osler en la endocarditis \\
\hline 1888 & Describió el edema angioneurótico hereditario \\
\hline \multirow[t]{6}{*}{1892} & Maniobra de Osler que se utiliza para detectar la pseudohipertensión \\
\hline & $\begin{array}{l}\text { Signo de Osler: presión arterial sistólica artificialmente elevada, debido a la calcificación aterosclerótica de las } \\
\text { paredes arteriales }\end{array}$ \\
\hline & Síndrome de Osler: cuadro clínico debido a la litiasis biliar en la ampolla de Vater \\
\hline & Síndrome de Osler: síndrome de vena cava inferior \\
\hline & Signo de Osler: pigmentación ocre en la esclerótica en la ocronosis \\
\hline & Tríada de Heschl-Osler: asociación de neumonía, endocarditis, y meningitis en un mismo paciente \\
\hline 1895 & Describió las complicaciones viscerales del LES \\
\hline 1897 & Describió la forma nodular del mixedema pretibial \\
\hline 1898 & Primera descripción del síndrome de Cushing \\
\hline 1899 & Primera descripción del síndrome de Banti \\
\hline 1900 & Primera descripción del síndrome Churg-Strauss \\
\hline 1901 & Describió la telangiectasia hemorrágica hereditaria o enfermedad de Rendu-Osler-Weber-Dimitri \\
\hline 1903 & Describió la policitemia rubra vera o enfermedad de Osler-Vaquez \\
\hline 1908 & Describió los nódulos de Osler en la endocarditis \\
\hline
\end{tabular}

LES: lupus eritematoso sistémico.

pacientes, lo que se conoce como enfermedad de Jaccoud-Osler-Libman, o sea la endocarditis bacteriana; junto a Sacks y a Libman describió a la endocarditis verrugosa en el lupus eritematoso sistémico, conocida como enfermedad de OslerLibman-Sacks ${ }^{14}$.

Describió en 1885, en la endocarditis, los llamados aneurismas micóticos, que se ubican principalmente en el territorio arterial cerebral. En la endocarditis describió la facie maligna lenta de Jaccoud-Osler que es el color blanco grisáceo o amarillo sucio de la cara junto a la seriedad trágica del paciente.

También se conoce como fenómeno de Osler a la aglutinación plaquetaria (que describió mientras estuvo en Londres). Con la denominación de síndrome de Osler también se conoce al síndrome de la vena cava inferior. Se denomina enfermedad de Rendu-Osler-Weber-Dimitri (también conocida como telangiectasia hemorrágica hereditaria), a una enfermedad con múltiples malformaciones vasculares en la piel y en las mucosas, además de fistulas pulmonares. Describió un parásito nematodo conocido como filaria de Osler. Se llama síndrome de Osler a los dolores cólicos con típica irradiación al dorso, acompañados de ictericia, escalofríos y fiebre intermitente, debidos a la litiasis biliar en la ampolla de Vater. Se conoce como enfermedad de Osler-Vaquez a la policitemia rubra vera. Signo de Osler se denomina a la pigmentación ocre en la esclerótica en la ocronosis. Se conoce como tríada de Heschl-Osler a la asociación de neumonía, endocarditis, y meningitis en un mismo paciente, por neumococo. Actualmente 
es conocida como tríada o enfermedad de Austrian, dado que Robert Austrian publicó 8 casos y revisión de la literatura en Archives of Internal Medicine en 1975.

Las manifestaciones clínicas del síndrome de Cushing son variables, y frecuentemente se confunden, lo que conlleva una demora en el diagnóstico y una elevada morbimortalidad. Harvey Cushing describió los signos y síntomas típicos de su síndrome, pero desafortunadamente atribuyó esas características al mixedema ${ }^{5}$. Posiblemente la primera descripción típica del síndrome de Cushing la realizó William Osler en 1898. Algunos autores llaman fenómeno de Osler a cuando existe demora en el diagnóstico del síndrome de Cushing ${ }^{15}$.

Al igual que con la endocarditis, describió magistralmente la angina de pecho (o enfermedad de Heberden $)^{16}$. El 15 de octubre de 1900, presentó en la reunión del Johns Hopkins Hospital Medical Society un caso de asma con cianosis, púrpura, mialgias, y eosinofilia. Es considerado por algunos autores como la primera descripción del síndrome Churg-Strauss ${ }^{17}$. Insinuó el probable papel que los agentes infecciosos podrían tener en la ateroesclerosis. Describió además una familia completa sugiriendo un patrón de herencia autosómica dominante en un subgrupo de pacientes con edema angioneurótico ${ }^{18}$.

Fue editor y fundador del Quarterly Journal of Medicine en 1908.

\section{Sus libros}

Osler fue un prolífico escritor: publicó más de 1.500 trabajos, entre artículos en revistas médicas, libros y monografías.

Su contribución a la literatura médica fue verdaderamente extraordinaria. El libro "The Principles and Practice of Medicine", aparecido en 1892, tuvo éxito entre los médicos de habla inglesa, y la primera edición se agotó rápidamente $(26.000$ ejemplares) ${ }^{19}$. Osler revisó y escribió sólo las primeras siete ediciones, y la séptima se publicó en 1909 (Figura 5). Su libro se tradujo al francés, al ruso, al alemán, al chino y al español.

En este libro el autor huye de dogmatismos, y se muestra casi siempre saludablemente escéptico. Fue de los primeros en señalar que el manejo de la apendicitis debe ser eminentemente quirúrgico ${ }^{20}$.
Otra obra impactante de su autoría es "Aequanimitas", cuya primera edición fue lanzada en $1904^{21}$. Al salir William Osler de la Universidad de Pennsylvania (Filadelfia) para Baltimore en 1889, dejó este mensaje a los estudiantes que se graduaban en ese momento "Aequanimitas es la calma, la serenidad, la ecuanimidad, y la transparencia que debe tener el médico ante cualquiera adversidad relacionada con el acto médico. Esto es realmente lo que necesitamos en nuestros países para poder reorganizar la estructura médica, en decadencia desde hace más de 100 años". En este texto, Osler recomienda a los estudiantes de medicina una lista de diez libros que deberían leer. El primero de ellos, la Biblia.

Escribió algunos ensayos sobre historia de la medicina, como "La evolución de la medicina moderna"22; "Los primeros libros médicos impresos"; "La medicina en la magna Inglaterra"; "El joven Laënnec"; "La evolución de la medicina interna"; "El crecimiento de la verdad"; "Las viejas humanidades"; "La nueva ciencia", y "Michael Servetus"3-6.

La grandeza de un personaje se traduce de varias maneras: en actos, pensamientos y palabras. En todas ellas, Osler dejó un recuerdo imborrable. Debido a su esfuerzo, el legado del altruista Johns Hopkins se convirtió, en buena parte gracias a él, en un vivero de clínicos, investigadores e innovadores, al pasar de un simple hospital a un centro de estudio y cultura de la más alta calidad.

\section{THE PRINCIPLES AND PRACTICE OF MEDICINE}

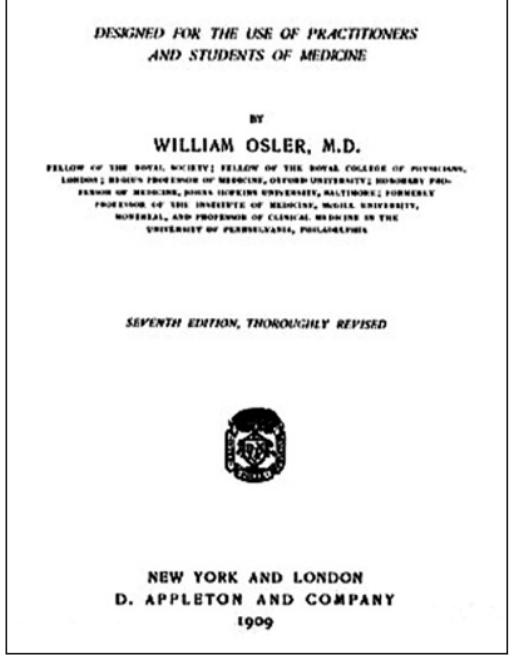

Figura 5. The Principles and Practice of Medicine, 1909. 
Uno de sus discípulos más importantes fue Harvey Cushing (1869-1939), quien en 1925 escribió su biografía "The life of Sir William Osler", que recibió, en 1926, el premio Pulitzer ${ }^{5}$. Esta biografía oficial, redactada por encargo de los discípulos y admiradores del maestro, más que un retrato crítico, constituye una verdadera hagiografía. Así, su grandeza se intuía desde niño y ninguno de sus actos puede empañarla. Esta visión fue indiscutida durante años, probablemente porque al indudable prestigio del biografiado se añadía el de su autor. No sorprende por tanto, que a su alrededor se haya creado una auténtica mística del personaje, inclusive se habla de él como el santo (Figura 6). Ensayos biográficos posteriores, especialmente el reciente libro de Michael Bliss, "William Osler. Una vida Entregada a la Medicina", han servido para acercarlo a un mundo real, en el que todos tenemos virtudes y defectos ${ }^{4,6}$.

El sucesor de Osler en el Johns Hopkins fue cuidadosamente seleccionado. Luego de un detenido análisis de los candidatos, el nombramiento recayó en Lewellyn F. Barker, quien había ingresado al Hospital años antes como profesor de Anatomía, y se destacaba como un profundo conocedor de las ciencias básicas. Es de advertir que el candidato de Osler era William S. Thayer (1864-1932), residente formado por él, y que se desempeñaba como Profesor Asociado de Medicina. Pero el Consejo Directivo, con el argumento de que era necesaria la presencia de un profesor que hiciera énfasis en las ciencias básicas en la enseñanza de la medicina, ratificó sus preferencias por Barker ${ }^{1-6}$.

Osler fue un gran coleccionista de libros de Historia de la Medicina. Después de su muerte su colección de más de 7.000 volúmenes formó la parte principal de la Biblioteca de Historia de la Medicina de la Universidad McGill, conocida como la librería Osleriana, que fue fundada en 1929.

\section{Sus aforismos}

El género aforístico es tan antiguo como la medicina occidental. La mayoría de los médicos argentinos recordamos los aforismos de Hipócrates de Cos (460-377 a.C.), especialmente los primeros trasmitidos por nuestros maestros "El Arte es largo, la vida es breve, la experiencia es falaz, el juicio difícil, la ocasión fugitiva". Recor-

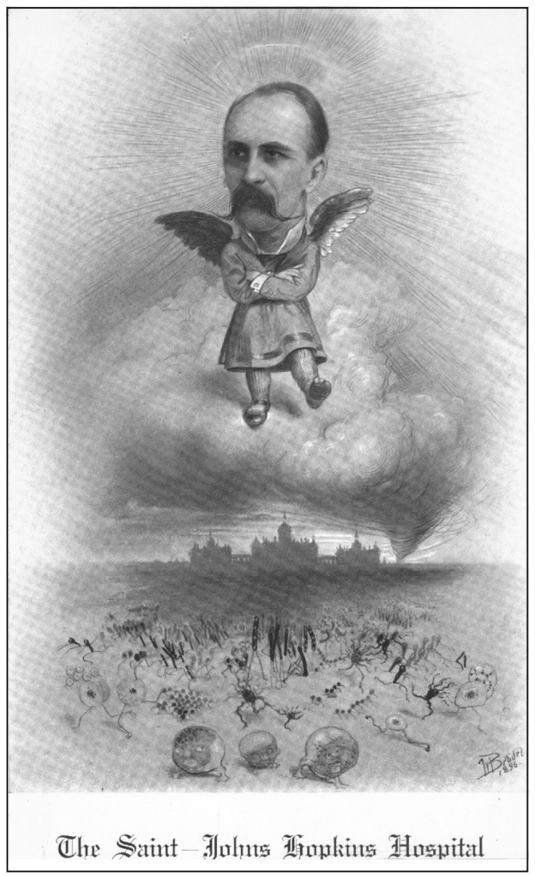

Figura 6. $\mathrm{El}$ santo.

damos los atribuidos entre otros al clínico francés Emile Charles Achard (1860-1944) "curar a veces, mejorar con frecuencia, consolar siempre”.

Osler enseñó la medicina interna en las salas del hospital, y en su recorrida mencionaba sentencias $\mathrm{y}$ aforismos vinculados al caso que se examinaba. Algunos de sus numerosos discípulos anotaron cuidadosamente este material ${ }^{23,24}$. Por ejemplo entre otras cosas decía que la neumonía era una enfermedad salvadora o el capitán del barco de la muerte que llevaba a la gente mayor hacia otra vida, o hacia la paz. Pregonaba que había que tener en cuenta el paciente individual más que las características especiales de la enfermedad. Indicó que "nosotros estamos en esta profesión por vocación, no por negocio; una vocación que exige constantemente auto-sacrificio, devoción, amor y ternura hacia sus semejantes. Al caer a un nivel puramente empresario, su influencia desaparece $y$ la auténtica luz de la vida se apaga. Deben trabajar con espíritu misionero, con un aliento de caridad para sobreponerse a las pequeñas envidias de la vida". También decía que "Ver enfermos (Práctica) sin leer libros (Teoría) es como navegar en el mar sin tener cartas de navegación, pero leer libros sin ver enfermos es como nunca navegar". Decía que "Ser buen médico significa poseer conocimientos 


\section{Tabla 2. Algunos de los Aforismos de William Osler 22,23}

- La educación es un proceso para toda la vida en el que el estudiante sólo puede hacer un comienzo durante sus estudios en la facultad

- He aprendido a ser un mejor estudiante y estar preparado para decir a mis alumnos "no lo sé"

- En lo que puede llamarse el método natural de enseñanza, el estudiante comienza con el paciente, continúa con el paciente y termina sus estudios con el paciente, utilizando libros y clases teóricas como herramientas, como medios para un fin

- Las clases teóricas superfluas causan bursitis isquiática

- Cuando uno observa a un médico examinar a su enfermo es fácil decir si ha tenido o no una enseñanza apropiada, y para este propósito quince minutos a la cabecera de la cama son preferibles a tres horas de escritorio

- Una gran universidad tiene una doble función, enseñar y pensar

- Por desechar el estudio de las humanidades, la profesión pierde una cualidad muy preciosa

- El sentido común en cuestiones médicas es raro y está en proporción inversa al grado de educación

- Los médicos buenos tratan las enfermedades, los médicos excelentes tratan a los pacientes que tienen enfermedades

- Un médico que se trata a si mismo tiene un estúpido por paciente

- En ninguna otra profesión es tan importante la cultura como en la medicina

- Siempre anote y registre lo inusual. Guarde y compare sus observaciones. Comunique o publique notas breves sobre lo que es llamativo o nuevo

- Nunca esconda el trabajo de otro debajo de su nombre

- Adquiera el arte de la imparcialidad, la virtud del método y la cualidad del rigorismo por sobretodo la gracia de la humildad

- El vicio mortífero del joven médico es la pereza intelectual

- Es sorprendente cómo con escasas lecturas un doctor puede practicar la medicina, pero no es sorprendente lo mal que puede hacerlo

- Cuando usted ha observado, lea. Y cuando pueda, lea las descripciones originales de los maestros quienes, con métodos rudimentarios de estudio, vieron tan claramente

- Con media hora de lectura en la cama cada noche como práctica constante, el hombre muy ocupado puede obtener buena educación antes que el plasma se establezca en los espacios periganglionares de la corteza gris

- Es más fácil comprar libros que leerlos, y es más fácil leerlos que absorberlos

- Lo que no le gusta que le hagan a usted, no lo haga a los otros

- Considere las virtudes de ser taciturno. Hable sólo cuando tenga algo que decir

- El silencio es un arma poderosa

- Aquel que sigue a otro no ve nada, no aprende nada y no busca nada

- Cuanto más grande es la ignorancia más grande es el dogmatismo

- Como dice Galeno, la confianza y la esperanza mejoran más que los fármacos. El que cura más es quien inspira más confianza

- La función principal del consultante es hacer un tacto rectal que usted ha omitido

- Uno de los primeros deberes del médico es educar a las masas para no tomar medicamentos

- La práctica de la medicina es un arte basado en una ciencia

- Una mujer con una lista escrita de sus síntomas: neurastenia

- El empiema necesita un cirujano y tres pulgadas de acero en lugar de un tonto como médico

- La pericarditis se diagnostica en proporción al cuidado del examen físico 
y tres H: Humanidad, Humor y Humildad". Por último entre otras cosas decía: "Observar, recopilar, clasificar y comunicar. Usad vuestros cinco sentidos. El arte de practicar la medicina sólo se aprende con la experiencia, no es una herencia, ni puede ser revelado. Aprended a ver, oír, palpar y oler; sabed que sólo merced a la práctica se puede llegar a ser un experto. La medicina se aprende al lado del enfermo y no en el aula. Mirar, razonar, comparar y controlar. Pero primero mirar. Dos ojos no ven igual la misma cosa, ni dos reflejos reflejan la misma imagen. Permitid que la palabra sea vuestra esclava, pero no vuestra dueña" "Vivid en la clínica". En la (Tabla 2) se listan algunos de sus aforismos más reconocidos ${ }^{24}$.

\section{A modo final}

Además de la angina de pecho que padeció desde 1902, Osler tuvo una neumonía en 1916, y varios procesos infecciosos bronquiales en 1918 y en 1919. En diciembre de 1918 se agravó de una infección pulmonar, y desarrolló un empiema que posteriormente se complicó con una rotura y hemorragia a la cavidad pleural. Su autopsia mostró varios abscesos en pleura y en el pulmón derecho, con una severa bronconeumonía. A nivel cardiovascular tenía una arterioesclerosis grave, con estenosis y calcificación importante de la arteria descendente anterior ${ }^{3-6}$. Su cuerpo fue velado en la catedral Christ Church en Oxford, y sobre su féretro se colocó su muy apreciado libro, Regio Medici. Su entierro se realizó el $1^{\circ}$ de enero de 1920. Su cuerpo fue cremado y sus cenizas reposan en la biblioteca Osler de la Universidad de McGill. Como filósofo, que también lo era, decía: "No deseo más epitafio que la mera inscripción en mi tumba, que enseñé a mis alumnos medicina en las salas del hospital". Su esposa murió nueve años después, y sus restos reposan al lado de su esposo. Al cumplir los 50 años de su fallecimiento, en 1969, el gobierno de Canadá lo honró con una estampilla (Figura 7).

En palabras del gran Francesco Petrarca (13041374), en su triunfo "Sobre la vida triunfa el amor, /sobre el amor, la muerte, /sobre la muerte, la fama, /sobre la fama, el tiempo, /y sobre el tiempo la eternidad", por sus aportes Osler está destinado al recuerdo eterno. Esperamos que la tradición osleriana nunca muera. Los médicos de este nuevo

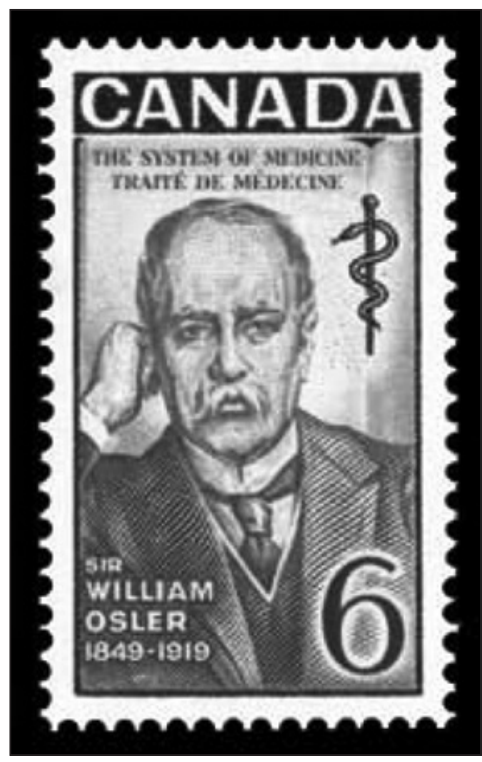

Figura 7. Estampilla.

milenio debemos mirar en este médico todo su ejemplo y enseñanza.

\section{Referencias}

1. Golden RL. William Osler at 150: An overview of a life. JAMA 1999; 282: 2252-8.

2. Barondess JA. Is Osler dead? Perspect Biol Med 2002; 45: 65-84.

3. Dubb A. William Osler re-visited. Adler Mus Bull 1999; 25: 14-9.

4. Bryan CS. Osler. Inspirations from a great physician. New York: Editorial Oxford University Press; 1997.

5. Cushing H. The Life of Sir William Osler. New York: Editorial Oxford University Press; 1940.

6. Bliss M. William Osler. A life in Medicine. New York: Editorial Oxford University Press; 1999.

7. Pasqualini RQ. Sir William Osler en McGill. Medicina (B Aires) 2005; 65: 471-4.

8. Belkin BM, Neelon FA. The art of observation: William Osler and the method of Zadig. Ann Intern Med 1992; 116: 863-6.

9. Lucey BP, Hutchins GM. Did Sir William Osler perform an autopsy at the Johns Hopkins Hospital? Arch Pathol Lab Med 2008; 132: 261-4.

10. Young P, Finn BC, Pellegrini D, Bruetman JE. [Hutchinson and his history]. Rev Med Chile 2010; 138: 383-7.

11. Starling PH. The case of Edward Revere Osler. J R Army Med Corps 2003; 149: 27-9. 
12. Golden RL. William Osler, urolithiasis, and God's own medicine. Urology 2009; 74: 517-21.

13. Al Rubaish AM. Gastric asthma: a clinical review. Saudi J Gastroenterol 2002; 8: 67-73.

14. Pruitt RD. William Osler and his Gulstonian Lectures on malignant endocarditis. Mayo Clin Proc 1982; 57: 4-9.

15. De P, Evans LM, Scanlon MF, Davies JS. “Osler's phenomenon”: misdiagnosing Cushing's syndrome. Postgrad Med J 2003; 79: 594-6.

16. Castagnino HE. [William Osler and angina pectoris: still prevailing concepts]. Arch Inst Cardiol Mex 1998; 68: 81-4.

17. Pearce JMS. Osler and the Churg-Strauss Syndrome. Eur Neurol 2007; 57: 185-7.
18. Osler W. Hereditary angioneurotic oedema. Am J Med Sci 1888; 95: 362-67.

19. Osler W. The Principles and Practice of Medicine. New York: Editorial Appleton; 1892.

20. Buzzi A. Historia del Tratado de Medicina Interna de William Osler. Medicina (B Aires) 2005; 65: 465-70.

21. Osler W. Aequanimitas. London: Editorial Lewis; 1948.

22. Osler W. The Evolution of Modern Medicine. New Haven: Editorial Yale University Press; 1923.

23. Bean WB. Sir William Osler's Aphorisms from his Bedside Teachings and Writings. New York: Editorial Achuman; 1950.

24. Buzzi A. Los aforismos de William Osler. Rev Asoc Méd Argent 2011; 124: 3-5. 